\title{
The Early Stages of the Development of Rolling Texture in Copper and Brass
}

\author{
T. LEFFERS and D. JUUL JENSEN \\ Metallurgy Department, Risø National Laboratory, DK-4000 Roskilde, Denmark
}

(Received November 8, 1987)

Dedicated to the memory of Professor Günter Wassermann

The texture development in copper and brass $(15 \%$ zinc) is followed by neutrondiffraction measurements from the early stage. The texture development is found to be different in the two materials from the very beginning (largely towards the respective final textures of copper and brass). As demonstrated by texture simulation starting with the experimental orientation distributions of the undeformed materials, the early difference in texture development is not a result of the differences in initial texture. The conclusion of the texture measurements-and of earlier microstructural observations confirmed in the present work-is that the development of the brass-type texture cannot be explained by any of the proposed twinning theories. The only existing model capable of reproducing the development of the brass texture at moderate strains without disagreement with experimental observations appears to be the Sachs model, or rather a modified Sachs model.

KEY WORDS: Rolling texture, ODF-analysis, neutron diffraction, copper, brass, twinning, Taylor model, Sachs model.

\section{INTRODUCTION}

In the sixties, when a number of different theories for the origin of the difference between the copper-type and the brass-type texture were discussed, it was considered an established fact that the early stages of development of the two types of texture were identicalup to reductions of about $50 \%$. The early texture development was 
normally quoted to be towards the final copper-type texture. In texture measurements by neutron diffraction Leffers and Kayworth (1973) and Tobisch and Mücklich (1974) observed clear differences between the textures of copper and brass at $40 \%$ and $30 \%$ reduction, respectively. It was argued against these observations that the observed early differences might be caused by differences in initial texture. Therefore one can still, also in recent literature (e.g. Proceedings of ICOTOM 8 , to be published) find the quotation that the early stage of texture development is the same for copper and brass.

In the present work we compare the early stages of texture development for various batches of copper and brass, and we supplement the texture measurements with texture simulations starting with orientation distributions corresponding to those of the applied materials before deformation (Leffers and Juul Jensen, 1986) - in order finally to settle the question whether there is a real difference between the copper and the brass texture from the early stage of development (we find that there is). We discuss the implications of these observations for the theories for f.c.c. rolling textures.

\section{EXPERIMENTAL}

\section{Materials}

The texture development was followed in two copper materials, copper I and copper II, and in one brass material, brass I. The materials were produced from copper of $99.98 \%$ purity and zinc of $99.99 \%$ purity by vacuum casting for copper and casting in pure argon for brass. The zinc content of the brass is $15 \%$ by weight. The cast material was subjected to various dual recrystallization processes with moderate rolling reductions followed by annealing in helium atmosphere at $550^{\circ} \mathrm{C}$. For instance copper II was rolled $49 \%$ and recrystallized, then rolled $20 \%$ and recrystallized. Copper I has a rather inhomogeneous grain-size distribution with an average grain size of $\sim 70 \mu \mathrm{m}$. Copper II has a less inhomogeneous grain-size distribution with an average grain size of $\sim 60 \mu \mathrm{m}$. Brass I has a homogeneous grain-size distribution with an average grain size of $\sim 40 \mu \mathrm{m}$. 
Apart from copper I, copper II and brass I specimens from the work of Leffers and Kayworth (1973) were included as copper III and brass II (with $15 \%$ zinc by weight as brass I). For these materials only one degree of reduction, $40 \%$, is represented. The materials had a rather uniform grain size of $80 \mu \mathrm{m}$ and $50 \mu \mathrm{m}$ for copper III and brass II, respectively. In all the grain-size measurements quoted annealing twins are neglected.

\section{Texture measurements}

The textures were measured by neutron diffraction at the DR3 reactor at Ris $\varnothing(\{111\},\{200\}$ and $\{220\}$ pole figures) and the ODFs were determined by the series expansion method as described by Juul Jensen et al. (1984). In general no ghost correction was made. For the lower degrees of reduction (with relatively weak textures) on which our interest is focused we considered this correction to be of marginal importance. For one material (brass II at $40 \%$ reduction) an ODF with ghost correction was made, and the difference from the uncorrected ODF was quite insignificant. Furthermore, the early-stage difference between the copper and the brass texture, which is the important observation, was seen in the pole figures as well as in the ODFs (see results section). The quarter pole figures to be quoted are averages of the four quarters of complete pole figures (corrected for absorption and extinction). In the preparation of specimens for texture measurement the surface material was removed from the two surfaces of the rolled plate (the thickness was reduced by $50 \%$ or more).

\section{TEXTURE SIMULATION}

Texture simulations, starting with orientation distributions corresponding to those of the experimental materials, were performed as described by the present authors (Leffers and Juul Jensen, 1986) - with minor improvements in the procedure for the transformation of the experimental ODF to a number of discrete orientations.

As described by Leffers and Juul Jensen (1986) some trends in the early copper textures are best simulated with a full-constraint Taylor model while others are best simulated with a relaxed 
constraint (lathe/chocolate bar) model - with a slight overall superiority for the relaxed-constraint model. In the present work we only quote results for the relaxed-constraint model, but the fullconstraint model would lead to exactly the same conclusion as far as the difference between the copper and the brass texture is concerned.

For the simulation of the brass textures we use a Sachs modelfor the simple reason that it works. The physical implications will be discussed later.

As in the work of Leffers and Juul Jensen (1986) random stresses are used to obtain the maximum degree of simularity between the simulated and the experimental textures. In terms of physics the random stresses simulate the interaction with the surrounding grains with their specific orientations, which is a random interaction when each grain is dealt with separately. In the present work (as in that of Leffers and Juul Jensen) the parameters of the random-stress procedure (the average level of random stress and the average strain range for which a given component of random stress is allowed to operate) are fixed by trial and error. However, the resulting random stresses are close to the average level of nonapplied stresses in a Taylor analysis $\dagger$, which appears to be the best fundamentally based estimate of the random-stress level. As to the question whether the early differences between the copper and the brass texture are caused by a difference in initial texture, the answer does not depend on the choice of parameters for the random-stress procedure.

\section{EXPERIMENTAL RESULTS}

Figure 1 shows the ODF development for copper I, copper II and brass I represented by the $\phi_{2}=45^{\circ}$ sections in Euler space. For copper II and brass I there is from the very beginning a continuous development towards the final copper and brass texture, respec-

† To be more specific: the average of the absolute values of the random-stress components is close to the average of the absolute values of the non-applied (misfit) stress components in a Taylor analysis - in both cases averaged over all strains (there is no work hardening in the model) and all grains. 


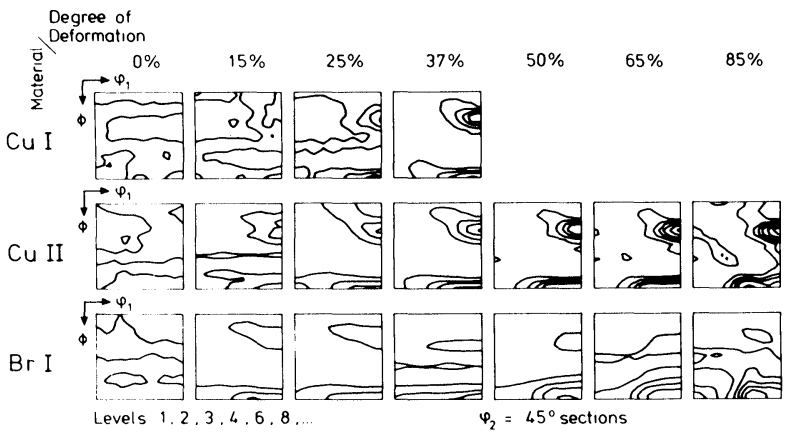

Figure 1 ODF development for $\mathrm{Cu}$ I, $\mathrm{Cu}$ II and Brass I.

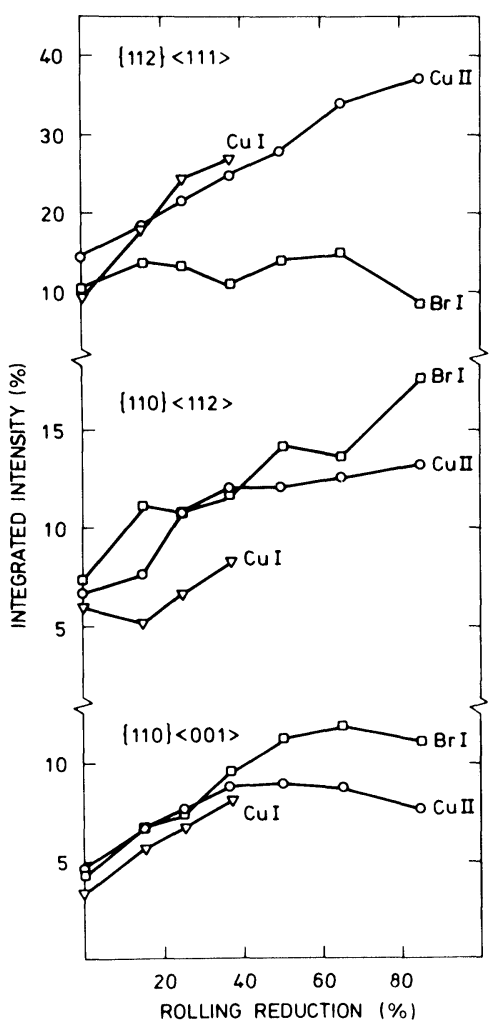

Figure 2 Development in three selected orientations for $\mathrm{Cu} \mathrm{I,} \mathrm{Cu} \mathrm{II} \mathrm{and} \mathrm{Brass} \mathrm{I.}$ 
(a)

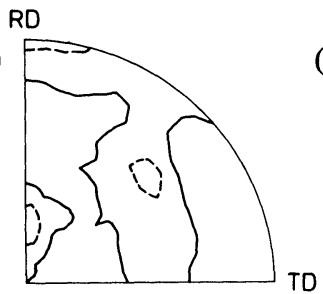

(b)

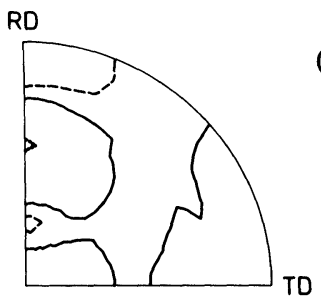

(c)

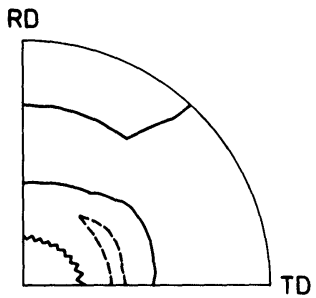

Figure $3\{200\}$ pole figures for $\mathrm{Cu}$ I (a), Cu II (b) and Brass I (c) after $25 \%$ reduction.

tively. For copper I this development is masked by the initial texture at $15 \%$ reduction, but it is clear already at $25 \%$ reduction.

In Figure 2 the texture development is represented by the development in orientation density for 3 characteristic orientations. The orientation densities are given as the integrated densities within $15^{\circ}$ in Euler space, but the result is basically independent of the exact way in which the development is monitored (integration within 5,10 or $15^{\circ}$ from the ideal orientations, density at the ideal orientations, peak density near the ideal orientations). It is clear that the specific copper orientation $\{112\}\langle 111\rangle$ develops strongest in copper from the very beginning (it never develops in brass).

(a)

$\mathrm{RD}$

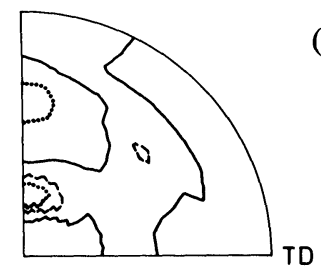

(b)

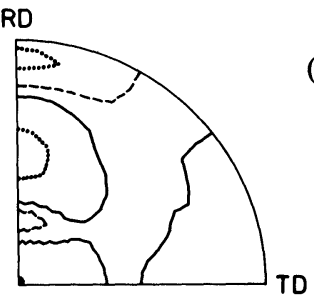

(c)

RD

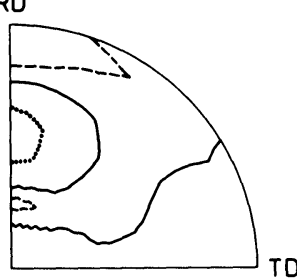

(d)

RD

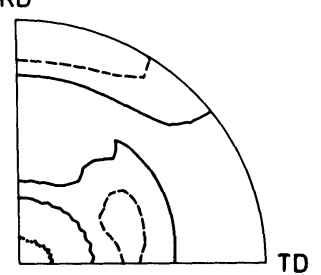

(e)

RD

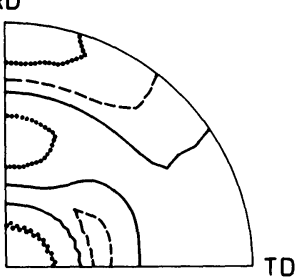

Figure $4\{200\}$ pole figures for Cu I (a), Cu II (b), Cu III (c), Brass I (d) and Brass II (e) after $37 / 40 \%$ reduction. 
(a)

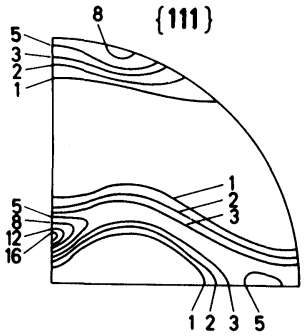

(c)

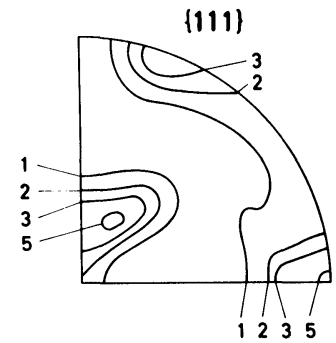

(b)

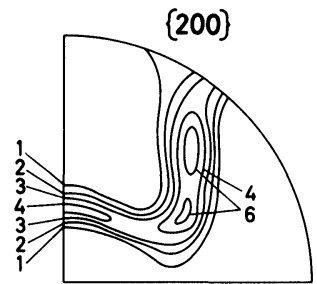

(d)

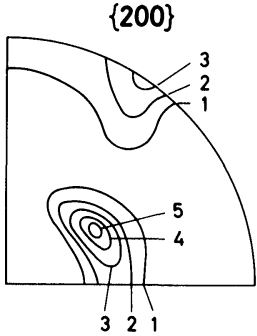

Figure $5\{111\}$ and $\{200\}$ pole figures for copper (a and b) and brass (c and d) after $95 \%$ reduction measured by $\mathrm{X}$-ray diffraction.

(a)

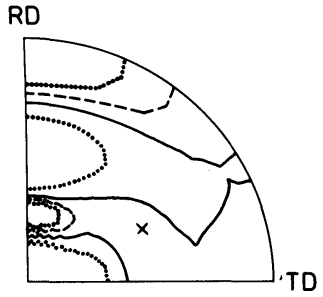

(c)

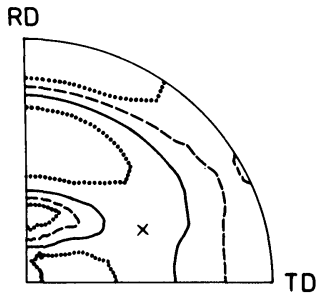

(b)

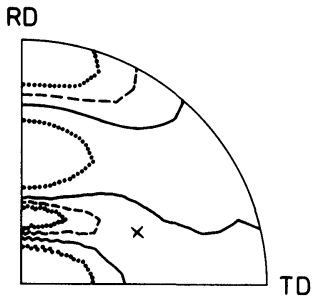

(d)

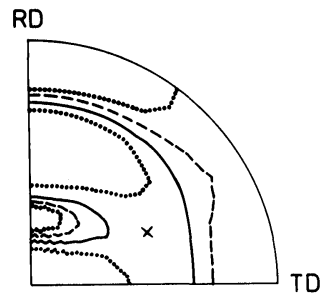

Figure $6\{111\}$ pole figure for $\mathrm{Cu}$ II(a), Cu III (b), Brass I (c) and Brass II (d) for $37 / 40 \%$ reduction (the $\{111\}$ pole figure for $\mathrm{Cu} \mathrm{I}$ has accidentally been erased from the computer file). 
The rest of the illustrations to be shown are pole figures. In all the pole figures (apart from the X-ray pole figures) the code for the pole-density contour lines is as follows: dotted curves correspond to pole densities 0.5 or 2.0 , full curves correpsond to pole densities 1.0 or 3.0 and dashed curves correspond to pole densities 1.5 or 4.0. It should be underlined that even though there are only three different curve symbols for six pole densities, the code is unambiguous (for instance on can identify the areas with lowest density, which is either below 0.5 or below 1.0 , and then count upwards).

In the pole figures the difference between copper and brass is most clearly visible for $\{200\}$. Figure 3 shows the $\{200\}$ pole figures for copper I, copper II and brass I for $25 \%$ reduction, and Figure 4 shows the $\{200\}$ pole figures for all five materials for $37 \% / 40 \%$ reduction. It is obvious that the copper and the brass pole figures in Figures 3 and 4 represent stages in the development towards the copper and brass pole figures for high reduction shown in Figure 5. At $37 \% / 40 \%$ reduction the texture difference is also visible in the $\{111\}$ pole figures as shown in Figure 6: for instance the copper pole figures have higher pole densities in the vicinity of the point marked with a cross than the brass pole figures. Comparison with Figure 5 shows that the $\{111\}$ copper and brass pole figures in Figure 6 also represent logical stages in the development towards the respective final pole figures.

\section{COMPARISON OF EXPERIMENT AND SIMULATION}

Figure 7 shows the simulated $\{200\}$ pole figures for relaxedconstraint Taylor and Sachs applied to the initial orientations of copper II and brass I for $25 \%$ reduction. It is very clear by comparison with Figure 3 that the type of the simulated texture (copper or brass) is determined by the model used in the simulation and not by the initial texture.

For all the materials in the present work it applies that the effect of the initial texture has practically vanished at $37 / 40 \%$ reduction. Figure 8 shows the simulated $\{111\}$ and $\{200\}$ pole figures for $37 \%$ reduction for the relaxed-constraint Taylor and the Sachs model starting with a random orientation distribution. The pole figures in 
THE EARLY STAGES OF ROLLING TEXTURES

475

Copper II
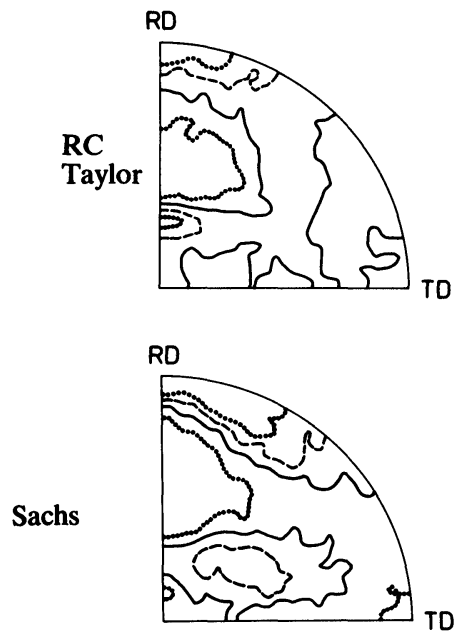

Brass I
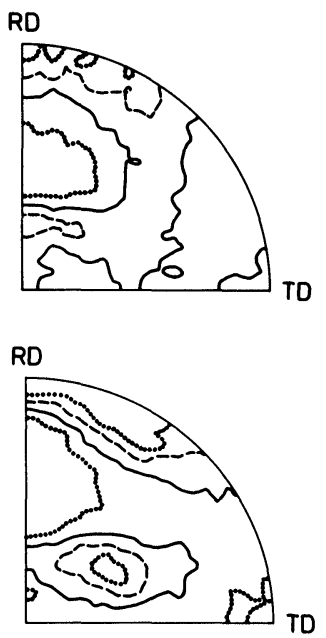

Figure 7 Simulated $\{200\}$ pole figures for $25 \%$ reduction of Copper II and Brass I using RC Taylor and Sachs.

$\{111\}$

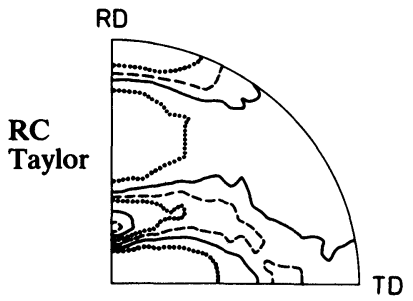

Sachs

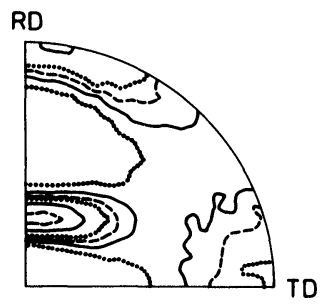

$\{200\}$
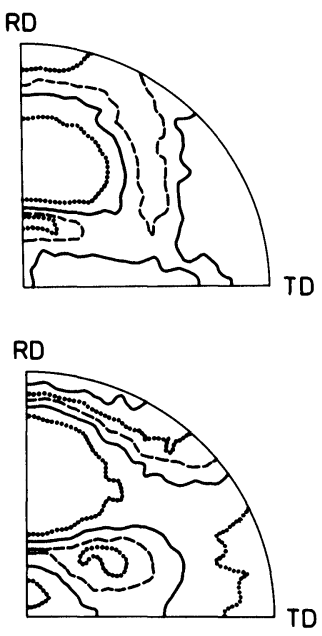

Figure 8 Simulated $\{111\}$ and $\{200\}$ pole figures for random initial texture, $37 \%$ reduction using RC Taylor and Sachs. 
Figure 8 are satisfactory simulations for all the experimental pole figures in Figures 4 and 6 (RC Taylor corresponding to copper and Sachs corresponding to brass).

\section{DISCUSSION}

Wassermann's original twinning theory for the brass-type texture (1963) as well as the more elaborate formulations by Kallend and Davies (1972) and Van Houtte (1978) presupposed that the initial texture development is towards the copper-type texture. The theory suggested that this development was interrupted at an intermediate stage because mechanical twinning transformed the orientations in one of the "beschränkten Fasertexturen" (orientations like $\{112\}$ $\langle 111\rangle)$ to orientations in the vicinity of $\{110\}\langle 001\rangle$, which would then change towards $\{110\}\langle 112\rangle$ with further deformation. The present work demonstrating that the development of the brass-type texture is different from that of the copper-type texture from the very early stage, obviously shows that the above assumptions in the twinning theory are not correct.

The twinning theory also referred to a volume effect of mechanical twins, i.e. it presupposed a substantial volume fraction of twinned material. As demonstrated by Leffers and Kayworth (1973) for intermediate strain and by Duggan, Hatherly, Hutchinson and Wakefield (1978) for higher strain the actually observed volume fraction of twinned material is too small to explain the brass-type texture as a volume effect of twins. This is illustrated in Figure 9 showing an example of the microstrucutre of brass I rolled to $37 \%$ reduction. Viewed in an arbitrary tilt the microstructure may appear to contain a significant fraction of bulky twins (estimated to about $10 \%$ by Leffers and Kayworth (1973)), but when the specimen is tilted so that the twin plane is approximately parallel to the electron beam, the apparently bulky twins turn out to consist of a great number of very thin twin lamellae. In brass I rolled $15 \%$ the major part of the material does not contain any twins. The twins observed are either single lamellae or very few lamellae grouped together as shown in Figure 10.

Having realized that the brass-type texture cannot be explained by a volume effect of mechanical twins, Duggan et al. (1978) 


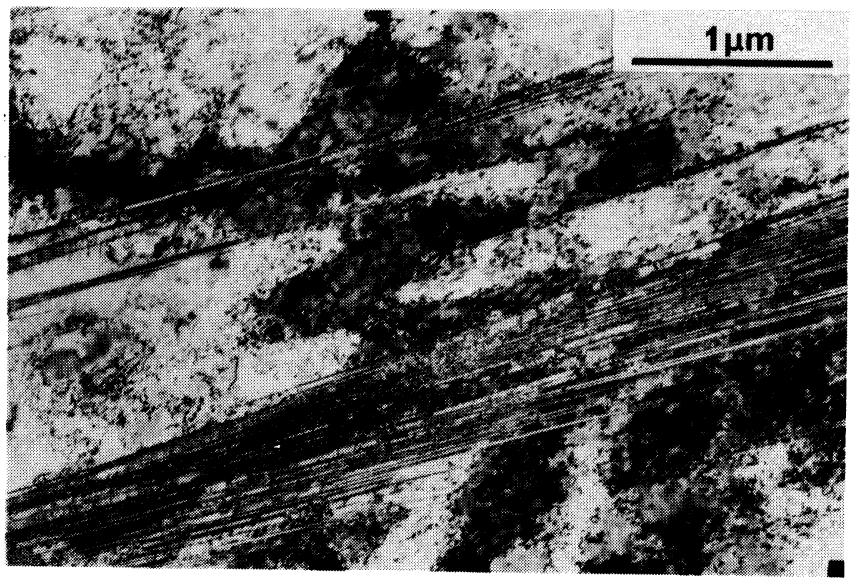

Figure 9. A bunch of thin twin lamellae in brass I rolled to $37 \%$ reduction.

suggested an alternative twinning theory in which the twins lead to the brass-type texture via their effect on the deformation pattern (and not directly via the orientation change they represent). The theory suggested that the twin lamellae on the primary slip plane make slip on intersecting slip planes more difficult and hence, via some kind of overshooting mechanism, lead to the formation of a strong $\{111\}\langle u v w\rangle$ texture peaking at reductions about $85 \%$.

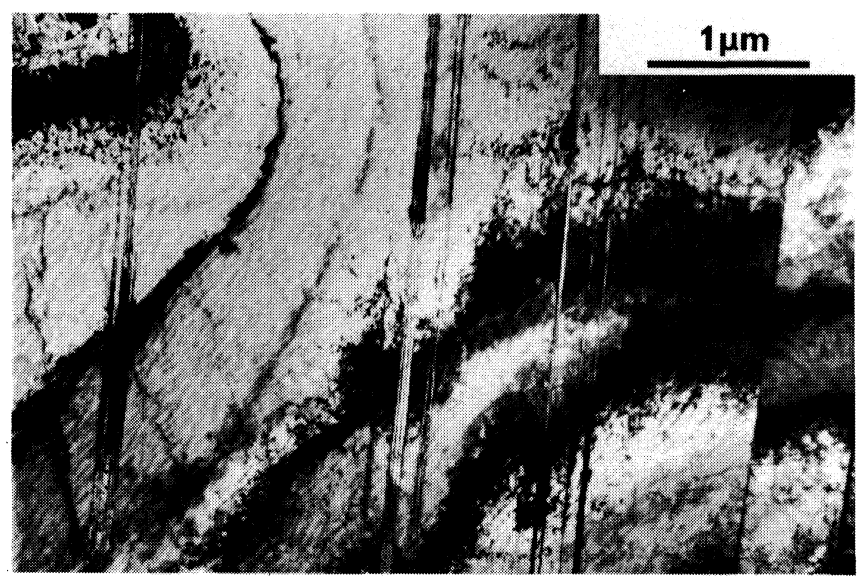

Figure 10 Scattered twin lamellae in Brass I rolled to $15 \%$ reduction. 
According to the theory the brass-type texture is formed by transformation of $\{111\}$ uvw $\rangle$ to $\{110\}\langle 001\rangle$ and $\{110\}\langle 112\rangle$ by shear banding and subsequent slip. This would mean that the formation of the brass-type texture takes place at a late stage, which is in obvious disagreement with the present observation of a continuous development towards the final brass-type texture from the very early stage of deformation. As argured by one of the present authors (Leffers, 1982) the development of the $\{111\}\langle\mathrm{uvw}\rangle$ texture component (with its peak at about $85 \%$ reduction) may be considered as a perturbation in the development of the brass-type texture (rather than the reason for its development). But, as we shall discuss later, it is a perturbation which affects the final brass-type texture.

The only existing model capable of reproducing the development of the brass-type texture (and doing so without disagreement with experimental observations) is the Sachs model - as suggested by one of the present authors (Leffers, 1968). The use of the Sachs model has been out of fashion in the last decades because the Taylor model has been considered much closer to reality. However, lately it is becoming increasingly accepted that the real world is more complex than this: we have to consider the whole range between the bounds defined by the Taylor and the Sachs model and not only the Taylor end of the range, e.g. Pedersen and Leffers (1987), Molinari et al. (1987), Canova (1988). The original Sachs model referred to a situation where the individual grains deform almost (but not completely, cf. Pedersen and Leffers (1987)) independently of the surrounding material. The situation referred to in our simulations with superimposed random stresses of a physically relevant magnitude is radically different (Pedersen and Leffers, 1987): there is interaction between the individual grains and the surrounding material, but the strictly deterministic Taylor interaction with a continuum matrix has been replaced by a stochastic interaction with neighbouring grains of unknown orientation. This presupposes that there is heterogeneous plastic interaction between the grains. As described by Pedersen and Leffers (1987) and Leffers (1988) the intragranular deformation heterogeneity observed experimentally in rolled brass does correspond to a level of plastic interaction which is sufficient to account for material continuity after deformation in accordance with the above Sachs concept. 
The simulated brass textures in Figures 7 and 8 do not only give a good qualitative simulation of the experimental brass texture; the numerical values of the pole densities are also fairly close to the experimental values. This quantitative agreement is not preserved at higher reductions (whereas it is preserved for simulated copper textures at high reduction (Leffers and Juul Jensen, 1986)): the simulated pole densities continue to increase with increasing reduction while the experimental pole densities tend to stabilize at a moderate level. This deviation must be due to the perturbations connected with the formation of the $\{111\}$ 〈uvw texture component which is not considered in the simulation. This also leads to a qualitative difference between the simulated and the experimental brass texture at higher reductions: the $\{111\}$ 〈uvw > component is absent in the simulated texture but visible in the experimental texture as seen in Figure 1.

We shall not enter into a detailed discussion of the mechanism which actually causes the change from the copper-type to the brass-type texture. It is obvious, however, that conditions which tie the glide dislocations to their slip plane and/or restrict the number of active slip planes are favourable for Sachs-type deformation (e.g. Pedersen and Leffers (1987)). Such conditions prevail in brass: low cross-slip frequency and presence of twin lamellae.

Neither shall we go into a detailed discussion of the formation of the copper-type texture. We just note that the experimental copper textures at low to moderate reductions are not full-constraint Taylor textures as one might expect since the deviation from equiaxed grain shape is not sufficient to apply the constraint relaxation of the standard relaxed-constraint model.

\section{Acknowledgement}

The authors want to thank B. Major for his participation in the initial stage of the present work.

\section{References}

Canova, G. R. (1988). To be published in Revue de Physique Appliquée.

Duggan, B. J., Hatherly, M. Hutchinson, W. B. and Wakefield, P. T. (1978). Met Sci., 12, 343-351.

Van Houtte, P. (1978). Acta Metall., 26, 591-604. 
Juul Jensen, D., Hansen, N., Kjems, J. K. and Leffers, T. (1984). Proc. 5th Risø Int. Symp., Microstructural Characterization of Materials by Non-Microscopical Techniques (edited by N. Hessel Andersen et al.), Risø Nat. Lab., Roskilde, Denmark, 325-332.

Kallend, J. S. and Davies, G. J. (1972). Phil. Mag., 25, 471-489.

Leffers, T. (1968). Ris $\emptyset$ Report No. 184, Danish Atomic Energy Commission.

Leffers, T. (1982). Textures of Materials (ICOTOM 6) (edited by S. Nagashima), The Iron and Steel Institute of Japan, Tokyo, 385-395.

Leffers, T. (1988). Proceedings of ICOTOM 8 (to be published).

Leffers, T. and Juul Jensen, D. (1986). Textures and Microstructures, 6, 231-263.

Leffers, T. and Kayworth, P. (1973). Proc. 3e Colloque Européen sur les Textures de Déformation et de Recristallisation des Métaux et leurs Applications Industrielles (ICOTOM 3), Société Française de Metallurgie, Nancy, 149-171.

Molinari, A., Canova, G. R. and Ahzi, S. (1987). Acta Metall., 35, 2983-2994.

Pedersen, O. B. and Leffers, T. (1987). Proc. 8th Risø Int. Symp., Constitutive Relations and Their Physical Basis (edited by S. I. Andersen et al.), Risø Nat. Lab., Roskilde, Denmark. 147-172.

Tobisch, J. and Mücklich, A. (1974). Texture, 1, 211-231.

Wassermann, G. (1963). Z. Metallkde., 54, 61-65. 